\title{
Estratégias de educação sexual a partir da percepção de estudantes de uma escola pública
}

\author{
Sex education strategies from the perception of students at a public school
}

\author{
Mayara Karolline Lima Ferreira dos Santos ${ }^{1}$, Luan de Araújo Queiroz ${ }^{1}$, Iara Luisy Silva Saltirio ${ }^{1}$, \\ Junnielly da Silva Costa Rios ${ }^{1}$, Hortência Maria Lopes de Souza Pinho ${ }^{1}$, Karwhory Wallas Lins da Silva ${ }^{2}$, \\ Thiago José Matos-Rocha ${ }^{3}$
}

\begin{abstract}
Resumo
O estudo teve como objetivo realizar estratégias de educação sexual a partir da percepção de estudantes de uma escola pública. Para tal, foi realizado um estudo quantitativo, descritivo, com 135 alunos do $1^{\circ}$ ao $3^{\circ}$ ano do ensino médio que responderam a um questionário estruturado composto por várias questões e com base nas respostas obtidas foram desenvolvidos métodos de intervenção em saúde, como palestras e oficinas. Dos estudantes que participaram da pesquisa, $17 \%$ eram do primeiro ano, $14 \%$ do segundo e $69 \%$ do terceiro ano. Observou-se que a iniciação sexual dos mesmos é precoce. $51 \%$ dos adolescentes eram do sexo masculino e a média de idade amostral foi de 19 anos. Mais de 90,0\% dos participantes $(n=123)$ referiram conhecer ao menos uma IST. Torna-se necessária a intervenção dos profissionais da saúde, articuladas com a escola e principalmente com a família proporcionando conversas, palestras e reflexões contemplando os temas Sexualidade e Saúde Reprodutiva.
\end{abstract}

Descritores: Estudantes, Educação em saúde, Doenças sexualmente transmissíveis, Sexualidade, Comportamento sexual, Adolescente

\section{Abstract}

The study aimed to carry out sex education strategies from the perception of students at a public school. To this end,

1. Graduado em Enfermagem pelo Centro Universitário CESMAC

2. Graduado em Farmácia e Biomedicina pelo Centro Universitário CESMAC

3. Professor de Parasitologia Humana da Universidade Estadual de Ciências da Saúde de Alagoas. Coordenador da Especialização de Análises Microbiológicas e Parasitológicas, Farmácia Hospitalar e Citologia Clínica no CESMAC

Trabalho realizado: Centro Universitário CESMAC

Endereço para Correspondência: Thiago José Matos Rocha. Rua Cônego Machado, 918 - Farol - 57051-160 Maceió - Alagoas Brasil. Tel.: +55 (82) 3215-5137. E-mail:thy_rocha@hotmail.com we conducted a quantitative study, descriptive, with 135 students from $1^{\circ}$ to $3^{\circ}$ year of high school who responded to a structured questionnaire composed of various issues and on the basis of the replies obtained were developed methods of intervention in health such as lectures and workshops. Of the students who participated in the research, $17 \%$ were in the first year, $14 \%$ of the second and $69 \%$ the third year. It was observed that the sexual initiation of same is premature. $51 \%$ of teens were male and the average age was 19 years sampling. More than $90.0 \%$ of participants $(n=123)$ reported knowing at least one STIs. Becomes necessary the intervention of the health professionals, articulated with the school and especially with the family providing conversations, lectures and musings contemplating themes sexuality and reproductive health.

Keywords: Students, Health Education, Sexually transmitted diseases, Sexuality, Sexual behavior, Adolescent

\section{Introdução}

A expressão Infecções Sexualmente Transmissíveis (ISTs) é usada para descrever as doenças que se disseminam principalmente pelo contato íntimo ${ }^{(1)}$. Englobam uma série de infecções causadas por vírus, fungos, protozoários e bactérias, cuja via preferencial de transmissão é a sexual ${ }^{(2)}$.

As ISTs são epidemias encontradas em todo o mundo e causam complicações biopsicossociais na vida de milhões de pessoas ${ }^{(1)}$. A adolescência é a fase da vida que traz mudanças anatômicas, fisiológicas, psicológicas, socioculturais e afetivas, sendo que neste período a sexualidade é enfrentada com grande intensidade, trazendo como consequência a iniciação da vida sexual ${ }^{(3)}$.

Além de todas as transformações biopsicossociais, a mídia e a facilidade de parceria sexual, estimulam esse público a se tornarem sexualmente ativos precocemente $^{(4)}$. A medida que eles iniciam a atividade sexual, se expõem à riscos como gravidez indesejada, HIV e outras ISTs. 
Alguns estudos publicados mostram que jovens e adolescentes são vistos como um grupo de alta vulnerabilidade às ISTs, questionando a necessidade de ações de educação em saúde que possibilitem a diminuição da transmissão dessas doenças nesse grupo etário ${ }^{(5-7)}$.

A educação em saúde é uma forma de disseminar as informações entre os indivíduos com o objetivo de prevenir doenças e/ou proteger e promover saúde ${ }^{(8)}$. Constitui-se como um recurso que permite fazer uma ligação entre o conhecimento científico de saúde e a população, produzindo mudanças no hábito de vida das pessoas, tornando-as mais independentes no conhecimento do processo saúde doença ${ }^{(9)}$.

A escola é um lugar importante para se trabalhar esses conhecimentos, as mudanças nos alunos podem ser diretas, através de alteração de comportamento em hábitos diários de curto ou longo prazo; ou indiretas, como divulgadores e fiscalizadores das atitudes de familiares, vizinhos e amigos. Portanto, é um local ideal para promover ações de educação em saúde ${ }^{(3)}$.

Em contrapartida, Azevedo et al (2012) ${ }^{(10)}$ referem que há uma lacuna de informações pela falta da educação sexual nas principais instituições em que os adolescentes convivem e, entre elas, destacam-se a escola e a família. As consequências disso são os sentimentos de culpa e de medo que atingem essa faixa etária, fazendo com que estes passem a buscar informações em fontes pouco seguras ou incapazes de ajudá-los ${ }^{(11)}$.

Uma pesquisa realizada por Reis, Oliveira-Monteiro (2007) ${ }^{(12)}$ com 100 adolescentes na faixa etária entre 14 e 19 anos constatou que as fontes mais citadas de informações sobre a sexualidade advêm dos colegas, primos e irmãos e apenas $10 \%$ das respostas referiam-se à escola como sendo uma fonte de informações sobre sexualidade. Dessa forma, essa pesquisa mostra que tantos os pais como a escola têm pouca participação como fontes de informações para os adolescentes. Alencar et al, $2008^{(9)}$ ressaltam que apesar do reconhecimento da importância do trabalho, este é pouco realizado no Brasil. Diante do exposto, o estudo teve como objetivo realizar estratégias de educação sexual a partir da percepção de estudantes de uma escola pública

\section{Material e Método}

\section{Tipo de estudo, local da pesquisa e amostra}

Foi realizado um estudo descritivo e transversal, realizado entre março e novembro de 2013, com 135 alunos (faixa etária de 17 a 19 anos), matriculados no $1^{\circ}$ ao $3^{\circ}$ ano do ensino médio na Escola Estadual Professora Irene Garrido, Maceió - AL. As interven- ções educativas foram desenvolvidas na escola com autorização da diretora escolar.

\section{Procedimentos e técnicas de coleta}

Inicialmente, foi solicitada à direção da escola a listagem de todos os alunos matriculados nas diversas séries. O trabalho de coleta dos dados e realização das intervenções educativas contou com a colaboração dos responsáveis pela área pedagógica, que facilitou o acesso dos pesquisadores aos estudantes dos dois turnos.

Para a coleta de dados, foi utilizado um questionário, elaborado para a obtenção dos dados necessários para o estudo, contendo perguntas sobre idade, sexo, sexualidade, métodos contraceptivos e ISTs. Os questionários foram não identificáveis para garantir o anonimato destes sendo utilizado um modelo utilizado em experiências anteriores por outros autores ${ }^{(13)}$. As seguintes perguntas do questionário contemplavam informações sobre Dados referente ao preservativo, Motivos de uso ou não do preservativo e por fim, sobre ISTs.

A pesquisa foi realizada em dois momentos: inicialmente, foram coletados dados através de um questionário de estudo realizado por Camargo e Ferrari (2009) ${ }^{(13)}$ para verificar o nível de conhecimento dos adolescentes; posteriormente, foram executadas oficinas de prevenção com grupos de dez a quinze estudantes, apenas para facilitar a realização das dinâmicas. As dinâmicas foram baseadas de acordo com o Manual do Multiplicador do Ministério da Saúde ${ }^{(14)}$. Os questionários foram respondidos em sala de aula e foram incluídos na pesquisa os estudantes presentes no momento da investigação e que constavam na lista de alunos.

\section{Métodos e técnicas de intervenção em saúde}

\section{Palestras}

As palestras foram feitas de forma dinâmica, utilizando cavalete e figuras para falar sobre as ISTs, formas de prevenção, transmissão e tratamento. Quanto à abordagem dos métodos contraceptivos, foi feita demonstração através do kit do Ministério da Saúde, com preservativo masculino e feminino, anticoncepcional oral e injetável, dispositivo intra-uterino (DIU) e diafragma, explicando o uso de cada um.

\section{Oficinas}

A primeira oficina abordou o tema sexualidade (práticas sexuais, desenvolvimento e transformação do corpo, o prazer e as relações de gênero). A segunda oficina abordou os métodos de prevenção das ISTs e HIV/AIDS, as formas de contágio e as repercussões 
biopsicossociais da infecção. Foram entregues aos alunos panfletos sobre o tema explicando as principais ISTs.

\section{Tratamento e organização das informações}

Os dados coletados foram submetidos ao programa Microsoft Excel ${ }^{\circledR}$ analisados em forma de tabelas.

\section{Aspectos Éticos}

A execução deste trabalho foi autorizada pelo Comitê de Ética e Pesquisa do Centro Universitário CESMAC, sob protocolo de número CAAE: 13453413.6.0000.0039.

\section{Resultados e Discussão}

Quanto ao perfil sociodemográfico, a maioria se declarou não branca $(n=65)$, católica $(n=55)$ e solteira (n=102). Mais de 65,0\% dos participantes afirmou já ter tido relação sexual com média de idade de início igual a 16 anos, sendo mais frequente a faixa entre 14 e 16 anos. As informações obtidas referentes ao uso do preservativo estão apresentadas nas tabelas 1 e 2 . Além das perguntas referidas na tabela, os participantes responderam uma pergunta relacionada à frequência do uso do preservativo e, dentre aqueles que a responderam ( $n=127), 13,4 \%$ e 8,7\% referiram usar de vez em quando e nunca usar o preservativo, respectivamente.

Embora os adolescentes detenham mais informações que os adultos, Clark et al, 2001(15) afirmam que esse conhecimento é baixo e que outros autores já demonstraram que eles praticam sexo oral e anal sem saber sobre essas fontes de contágio. Sabe-se que em cada adolescente existe uma forma individual de lidar com a sexualidade, mas é extremamente importante que eles possuam hábitos de proteção e que esses hábitos sejam parte integrante do erotismo ${ }^{(16)}$.

Conforme a Tabela 2, 45,8\% dos alunos responderam que sempre utilizam camisinha durante as relações sexuais, principalmente para evitar a gravidez.

\section{Tabela 1}

Dados referentes ao uso de preservativo relatado pelos adolescentes de uma escola pública, no período de março e novembro de 2013

\begin{tabular}{|c|c|c|c|c|c|}
\hline \multirow{3}{*}{ Dados referentes ao preservativo } & \multicolumn{4}{|c|}{ Resposta } & \multirow{3}{*}{ Missings } \\
\hline & \multicolumn{2}{|c|}{ Sim } & \multicolumn{2}{|c|}{ Não } & \\
\hline & $\bar{N}$ & $\% *$ & $N$ & $\% *$ & \\
\hline Uso na primeira relação sexual & 66 & 54,5 & 55 & 45,5 & 14 \\
\hline Uso na última relação sexual & 64 & 56,2 & 50 & 43,8 & 21 \\
\hline Diminui o prazer da relação sexual & 57 & 51,3 & 54 & 48,7 & 24 \\
\hline Devem usá-lo em todas as relações sexuais & 117 & 91,4 & 11 & 8,6 & 7 \\
\hline $\begin{array}{l}\text { Caso o(a) parceiro(a) não quiser usar a camisinha, transaria } \\
\text { mesmo assim }\end{array}$ & 32 & 24,8 & 97 & 75,2 & 6 \\
\hline
\end{tabular}

*As porcentagens foram calculadas em relação ao total de respostas válidas (desconsiderando os missings).

\section{Tabela 2}

Informações adicionais obtidas acerca do uso da camisinha relatado pelos adolescentes de uma escola pública, no período de março e novembro de 2013

\begin{tabular}{lcc}
\multicolumn{1}{c}{ Motivos de uso ou não do preservativo } & \multicolumn{2}{c}{ Respostas } \\
& $N \%^{*}$ & 2,8 \\
Nunca usei & 3 & 0,9 \\
Não uso, porque não sei como usar. & 1 & 5,6 \\
Não uso porque não gosto & 3 & 2,8 \\
Não uso, porque não confio nele & 1 & 0,9 \\
Não uso, porque não sei como conseguir a camisinha & 14 & 13,1 \\
Uso apenas quando transo com algumas pessoas, para evitar DST & 30 & 28,0 \\
Uso para evitar gravidez & 49 & 45,8 \\
Sempre uso nas minhas relações sexuais. & 28 \\
\hline
\end{tabular}

*As porcentagens foram calculadas em relação ao total de respostas válidas (desconsiderando os missings). 
Moura et al, 2011 ${ }^{(17)}$ afirmam que o uso de preservativo masculino é o método mais difundido entre as pessoas sexualmente ativas, confere dupla proteção, previne a gravidez, AIDS e outras ISTs. As principais causas de sua não utilização são: a confiança no parceiro e ocorrência de imprevisibilidade das relações sexuais.

Um estudo que avaliou o conhecimento sobre IST/ AIDS por estudantes adolescentes verificou a maior parte das meninas $(75 \%)$ e dos meninos (52\%) adquiriam informações da televisão e em segundo lugar (65\% e $42 \%)$, respectivamente, tiravam as dúvidas com os professores.

Mais de $90,0 \%$ dos participantes $(n=123)$ referiram conhecer ao menos uma IST. As informações referentes ao conhecimento dos participantes acerca das DSTs estão apresentadas na tabela 2 . Vale considerar ainda nesse contexto que quase metade dos alunos $(49,6 \%$; $\mathrm{n}=58$, considerando apenas os que responderam essa pergunta) não sabia se o fato de ter uma IST aumentaria as chances de se pegar outra IST e o vírus HIV e pouco mais de $10,0 \%(n=12$, considerando somente aqueles que responderam essa pergunta) afirmou que o adolescente portador do vírus HIV não deveria continuar indo à escola.

A educação sexual pode ser atribuída a cultura, religião e origem familiar, muitos cuidados de saúde, sejam curativos ou preventivos são adquiridos e reproduzidos dos antecedentes. Com esse hábito, muitas informações importantes e verídicas deixam de ser passadas, sendo fundamental a execução de pesquisas que busquem conscientizar os adolescentes dos riscos, apresentando as estratégias de prevenção disponíveis ${ }^{(18)}$.

O público se mostrou participativo durante as ações de intervenção. Durante as oficinas os adolescentes estavam à procura de informações sobre o uso de preservativos, interagindo constantemente:

"Acho que nós meninas devemos estar mais atentas quanto ao uso de camisinhas e carregar na bolsa quando for sair, até porque, a gravidez é mais complicada para gente do que para os meninos..." (menina, durante oficina).

"Temos que se ligar, uma criança necessita de dinheiro e cuidados..." (menino, durante oficina).

Durante as oficinas de sexualidade e contracepção, verificou-se que os alunos não se encontravam constrangidos falando de forma espontânea sobre todos os temas abordados; neste momento foram entregues aos alunos panfletos explicando a respeito das principais ISTs, os estudantes fizeram perguntas sobre o conteúdo dos folhetos.

Em abordagem semelhante, no estudo realizado por Moura et al (2011) ${ }^{(17)}$ realizado para descrever as fontes de informação sobre sexualidade e contracepção utilizadas por adolescentes mostrou que mais de $85 \%$ das adolescentes possuíam alguma informação sobre como evitar filhos e IST antes de engravidar. Cerca de $55 \%$ afirmou ter alguém com quem conversar sobre o assunto, e na escala de preferencias ficaram as amigas com $36,3 \%$, seguida da mãe com $25,5 \%$ e parceiro com $16,6 \%$. As adolescentes em geral sabem que o preservativo evita doenças e gravidez, mas sentem dificuldades em usar.

Por outro lado, o estudo realizado por Rolim et al $(2016)^{(19)}$ A grande maioria dos adolescentes não tem conhecimento de programas/oficinas de educação sexual e prevenção da Aids na escola e no município. Dos que conhecem os programas, $80.6 \%$ não frequentam no município e a metade não participa na escola. Poucos adolescentes obtêm camisinha, informativos, testes para Aids e pílulas anticoncepcionais dos postos de saúde. A maioria dos adolescentes $(78,4 \%)$ gostaria de aprender mais sobre sexualidade, gravidez e Aids. Esses resultados apontam a necessidade dos órgãos públicos tornarem mais acessíveis os programas de educação sexual nas escolas e especialmente nos serviços de saúde.

Também foi ministrada uma palestra educativa sobre métodos contraceptivos com um kit demonstrativo do Ministério da Saúde, eles relataram gostar das atividades, que foi um aprendizado que eles que irão fazer uso e comentaram sobre a necessidade de promover educação sexual na escola.

Figueiredo, Ayres (2002) ${ }^{(18)}$ destacam a importância de conscientizar a população com atividades de intervenção e estar sensível as necessidades de cada público para orientá-los e promover transformações benéficas a comunidade.

Assim como os familiares, a escola também enfrenta problemas para tratar sobre sexualidade, mas é evidente a necessidade de discutir o tema em sala de aula, já que o fornecimento de assistência, saúde e educação é escasso na sociedade ${ }^{(4)}$.

Na pesquisa realizada por Almeida et al (2017) ${ }^{(20)}$ com 22 adolescentes entre 16 e 19 anos de idade, estudantes do Ensino Médio de uma escola pública, analisaram a sexualidade, educação sexual; compreensão de comportamentos de risco e os resultados revelaram a necessidade de ações educativas de prevenção para os adolescentes, pois a falta de informações contribui para a sua vulnerabilidade. Os jovens reconheceram a importância da educação sexual e a implementação de estratégias de promoção e de proteção a saúde no ambiente escolar para contribuir e fortalecer o autocuidado.

\section{Conclusão}

De acordo com os resultados apresentados, os estudantes afirmaram ter conhecimento sobre as IST's e 
Resultados referentes às perguntas relacionadas às ISTs relatado pelos adolescentes de uma escola pública, no período de março e novembro de 2013

\section{O que significa Infecções Sexualmente Transmissíveis (IST's)?}

\section{Respostas} N

Não sei

São doenças que se pega principalmente pelo sexo

$5 \quad 3,7$

$\% *$

É uma doença venérea, da rua, do mundo, e que se pega apenas com profissionais do sexo (como, por exemplo, prostitutas)

Quem pode pegar uma IST?

Não sei

Qualquer pessoa que tenha relação sexual com mais de um parceiro, sem proteção da camisinha

Somente aqueles que frequentam casas noturnas (como por exemplo, cabarés e casas de shows eróticos) e fazem sexo com várias pessoas

Qualquer pessoa, cujo companheiro ou companheira sexual tenha relações sexuais desprotegidas (sem camisinha)

\section{Para que serve a camisinha?}

Não sei

Para evitar as doenças venéreas (as IST's)

127

3,7

7

Para proteger do vírus da Aids (o HIV)

$\mathrm{N} \quad \% *$

7

5,2

$69 \quad 51,1$

64,4

Para evitar gravidez

\begin{tabular}{|c|c|c|}
\hline Quais das infecção vaginal/ISTs abaixo você conhece? & $N$ & $\% *$ \\
\hline Sífilis & 91 & 67,4 \\
\hline Cancro mole & 25 & 18,5 \\
\hline Condiloma acuminado & 48 & 35,6 \\
\hline Gonorreia & 68 & 50,4 \\
\hline Tricomoníase & 35 & 25,9 \\
\hline AIDS/HIV & 76 & 56,3 \\
\hline Outras & 33 & 24,4 \\
\hline Onde você recebe (eu) informação sobre IST's? & $N$ & $\% *$ \\
\hline Por intermédio de Agente comunitários de Saúde & 18 & 13,3 \\
\hline No centro de saúde & 26 & 19,3 \\
\hline Pelo Rádio & 12 & 8,9 \\
\hline Pela televisão & 62 & 45,9 \\
\hline Pelo jornal & 26 & 19,3 \\
\hline Lendo material informativo & 36 & 26,7 \\
\hline Pela igreja & 12 & 8,9 \\
\hline Pela escola & 85 & 63,0 \\
\hline No trabalho & 12 & 8,9 \\
\hline Na Associação comunitária & 2 & 1,5 \\
\hline Com os agentes de saúde & 4 & 3,0 \\
\hline Na farmácia & 47 & 35,1 \\
\hline No supermercado & 23 & 17,2 \\
\hline Com pessoas de organizações Não-governamentais (ONGs) & 1 & 0,7 \\
\hline Em outra fonte. & 5 & 3,7 \\
\hline
\end{tabular}

*As porcentagens foram calculadas em relação ao total de participantes considerando que nessa sessão os mesmos poderiam assinalar mais de uma alternativa. 
a importância do uso de preservativos como proteção.

Concluiu-se dessa forma ser necessária a intervenção dos profissionais da saúde, articuladas com a escola e principalmente com família para esclarecimento dos jovens, baseada na participação em conversas e reflexões sobre os temas da Sexualidade e de Saúde Reprodutiva, bem como no questionamento de valores que discriminam pessoas portadoras de doenças sexualmente transmissíveis.

\section{Referências}

1. Pinto RJ, Fernandes AI, Mesquita C, Maia AC. Childhood adversity among institutionalized male juvenile offenders and other high-risk groups without offense records in Portugal. Violence Vict. 2015; 30(4):600-14.

2. Farid NDN, Rus CS, Dahlui M, Al-Sadat N, Aziz NA. Predictors of sexual risk behaviour among adolescents from welfare institutions in Malaysia: a cross sectional study. BMC Public Health. 2014; 14(Suppl 3):S9.

3. Ahorlu CK, Pfeiffer C, Obrist B. Socio-cultural and economic factors influencing adolescents' resilience against the threat of teenage pregnancy: a cross-sectional survey in Accra, Ghana. Reprod Health. 2015; 12(1):1.

4. Macintyre AKJ, Vega ARM, Sagbakken M. From disease to desire pleasure to the pill: a qualitative study of adolescent learning about sexual health and sexuality in Chile. BMC Public Health. 2015; 15(1):945.

5. Camilo VMB, Freitas FLS, Cunha VM, Castro RK, Sherlock MSM, Pinheiro PNC, et al. Educação em saúde sobre DST/ AIDS com adolescentes de uma escola pública, utilizando a tecnologia educacional como instrumento. DST J Bras Doenças Sex Transm. 2009; 21(3): 124-8.

6. Oliveira DC, Gomes AMT, Pontes APM, Ribeiro MCM. Conhecimentos e práticas de adolescentes acerca das DST/HIV/ AIDS em duas escolas públicas municipais do Rio de Janeiro. Esc Anna Nery Rev Enferm. 2009; 13(4):833-41.

7. Coelho RFS, Souto TG, Soares LR, Lacerda LCM, Matão MEL. Conhecimentos e crenças sobre doenças sexualmente transmissíveis e HIV / AIDS entre adolescentes e jovens de escolas públicas estaduais da região oeste de Goiânia. Rev Patol Trop. 2011; 40(1):56-66.
8. Malta DC, Silva MAL, Mello FCM, Monteiro RA, Porto DL, Sardinha LMV, et al. Saúde sexual dos adolescentes segundo a Pesquisa Nacional de Saúde dos Escolares (PeNSE). Rev Bras Epidemiol. 2011;14(1 Supl 1):147-56.

9. Alencar RA, Silva L, Silva FA, Diniz RES. Desenvolvimento de uma proposta de educação sexual para adolescentes. Ciênc Educ (Bauru). 2008; 14(1):159-68.

10. Azevedo E, Pelicioni MCF, Westphal MF. Práticas intersetoriais nas políticas públicas de promoção de saúde. Physis (Rio de J). 2012; 22(4):1333-56.

11. Moura JRA, Figueiredo IGA, Santos TNC, Sousa EC, Vieira TF, Lima SEA. Conversas de adolescentes sobre drogas e sexualidade: um relato de experiência. Revinter. 2015;8(2):117-30.

12. Reis AOA, Oliveira-Monteiro NR. Sexualidade e procriação na ótica de jovens de periferias sociais e urbanas. Rev Bras Crescimento Desenvolv Hum. 2007; 17(2):54-63.

13. Camargo EAI, Ferrari RAP. Adolescentes: conhecimentos sobre sexualidade antes e após a participação em oficinas de prevenção. Ciênc Saúde Coletiva. 2009; 14(3): 937-46.

14. Brasil. Ministério da Saúde. Coordenação Nacional de DST e Aids. Manual do multiplicador: adolescente. Brasília: Ministério da Saúde; 2000. 160p.

15. Clark LR, Jackson M, Allen-Taylor L. Adolescent knowledge about sexually

transmitted diseases. Sex Transm Dis. 2002; 29(8):436-43.

16. Brêtas JRS, Ohara CVS, Jardim DP, Muroya RL. Conhecimento sobre DST / AIDS por estudantes adolescentes. Rev Esc Enferm USP. 2009; 43(3):551-7.

17. Moura LNB, Gomes KRO, Rodrigues MTP, Oliveira DC. Informação sobre contracepção e sexualidade entre adolescentes que vivenciaram uma gravidez. Acta Paul Enferm. 2011; 24(3):320-6.

18. Figueiredo R, Ayres JRCM. Intervenção comunitária e redução da vulnerabilidade de mulheres às DST/ Aids em São Paulo, SP. Rev Saúde Pública. 2002; 36(4): 96-107.

19. Rolim SR, Bielenki CRZ, Béria JU, Schermann LB, Santos AMPV, Arossi GA. Conhecimento e acesso aos programas de educação sexual e prevenção da Aids: um estudo com adolescentes escolares. Aletheia, 2016; 49(2):110-21.

20. Almeida RAAS, Corrêa RGCF, Rolim ILTP, Hora JM, Linard AG, Coutinho NPS, et al. Conhecimento de adolescentes relacionados às doenças sexualmente transmissíveis e gravidez. Rev Bras Enferm. 2017;70(5):1087-94.

Trabalho recebido: $27 / 12 / 2017$

Trabalho aprovado: $31 / 07 / 2018$ 